\title{
Universidad y cambio de modelo productivo audio- visual: el proyecto CIAKL (Cinema and Industry Alliance for Knowledge and Learning)
}

\author{
Juan Manuel CORBACHO VALENCIA \\ Universidad de Vigo \\ jmcorbacho@uvigo.es \\ Oswaldo García CRESPO \\ Universidad de Vigo \\ oswaldogarcia@uvigo.es \\ Alberto DAFOnTE GómeZ \\ Universidad de Vigo \\ albertodafonte@uvigo.es
}

\section{Resumen}

El sector audiovisual está inmerso en un proceso de cambio de modelo productivo más allá de la coyuntura económica del último lustro. En este contexto, emerge el proyecto europeo CIAKL como alternativa de formación para estudiantes de cinco universidades europeas que ofrecen estudio de grado en comunicación audiovisual. Se explora una experiencia pionera en este ámbito desde su faceta docente hasta la práctica, siguiendo el proceso que recorre la idea de negocio desde su origen hasta su implementación.

Palabras Clave: Proyecto europeo, industrias culturales, ideas de negocio, modelo productivo audiovisual.

University and the change of audiovisual production model: the European project CIAKL (Cinema and Industry Alliance for Knowledge and Learning)

\begin{abstract}
The audiovisual sector is undergoing a sound change in its production models beneath economic crisis. This is were the European project CIAKL comes into play as education alternative for students of five European universities offering film studies. This paper explores this pioneering initiative from its teaching feature to the implementation following the process of creating and developing a business idea into a working project.
\end{abstract}

Key Words: European project, cultural industries, business idea, audiovisual production model.

\section{Referencia normalizada:}

Corbaho Valencia, J. M.; García Crespo, O.; Dafonte Gómez, A. (2013) Universidad y cambio de modelo productivo audiovisual: el proyecto CIAKL (Cinema and Industry Alliance for Knkowledge and Learning). Historia y Comunicación Social. Vol. 13 N Especial Octubre. Págs. 243-253

\section{Sumario}

1. El proyecto CIAKL y su contexto. 2. Metodología. 3. Filosofía y objetivos. 4. El curso. 4.1. Identificación de necesidades. 4.2. El diseño de contenidos. 4.3 Metodología y herramientas. 4.4. Implementación del programa docente. 5. De la idea al proyecto "Creactivos". 5.1. El desarrollo del proyecto "Creactivos". 6. Conclusiones. 7. Referencias bibliográficas. 8. Notas. 


\section{EI proyecto CIAKL y su contexto}

Las últimas décadas han estado marcadas, para la enseñanza universitaria, por un proceso de revisión de su papel en la sociedad, del modelo educativo que ofrecen y de las metodologías docentes que venían aplicando, sin grandes cambios, prácticamente desde sus orígenes.

El papel de la educación universitaria en la formación del individuo y la relación de este proceso con la capacitación para su entrada en el mercado laboral, se ha venido cuestionando de manera insistente en lo últimos años, con la crítica recurrente a la distancia entre "teoría" y "práctica profesional" en las enseñanzas universitarias; una crítica que se recrudece en una época en la que los recién titulados se encuentran con un mercado laboral que no tiene hueco para ellos.

Por otra parte, en los últimos años hemos venido asistiendo también a otro fenómeno que, de forma paulatina, puede operar un gran cambio en el modelo educativo de nivel universitario y que está relacionado con la enseñanza no presencial. La enseñanza a distancia cuenta ya con una larga trayectoria que las herramientas de teledocencia ligadas a internet habían contribuido a reforzar, pero su modelo educativo es, generalmente, la adaptación de los patrones de la formación universitaria presencial a un contexto en el que las relaciones docente-discente se establecen a distancia, por lo que no representa una revolución en este sentido. Sin embargo, es la experiencia de la formación a distancia convencional la que da lugar a nuevos modelos más rupturistas y relacionados con el libre acceso de la sociedad a los contenidos formativos que las instituciones universitarias elaboran, como la iniciativa puesta en práctica por el MIT en 2001 de poner a disposición de cualquier usuario interesado materiales docentes empleados en los cursos impartidos en la institución (OpenCourseWare) con la finalidad única de potenciar la sociedad del conocimiento y con la posibilidad añadida de ser reutilizados. Esta iniciativa acabaría desembocando en 2005 en la creación del OpenCourseWare Consortium para agrupar a todas aquellas universidades -entre ellas varias españolas- interesadas en ofrecer materiales docentes bajo esta modalidad.

La revisión de estas primeras experiencias de recursos educativos abiertos nos lleva al surgimiento de los MOOCs (Massive Open Online Courses) con algunos puntos en común con estas iniciativas pioneras, pero también con notables diferencias a pesar del amplio abanico de posibilidades que presentan. El término MOOC, atribuido a Cormier ${ }^{1}$ por un lado y Alexander ${ }^{2}$, por otro, para referirse al que se considera primer curso de estas características "Connectivism and Connective Knowledge - CCK08", de la Universidad de Manitoba, impartido en 2008, hace referencia a un tipo de curso organizado desde una universidad que responde a una estructura formativa reglada y evaluable a través de pruebas, que se desarrolla de forma no presencial a través de internet, con acceso libre a los materiales formativos (aunque generalmente se paga por el título que acredita la superación del curso), de matrícula abierta y sin límite de alumnado inscrito. A pesar de que se pueden establecer categorías más concretas dentro de este tipo de curso (SCOPEO, 2013: 10-22) la base del modelo, que está 
experimentando un enorme crecimiento ${ }^{3}$ a nivel global desde sus primeros pasos en 2008, es la expuesta.

En el contexto que acabamos de presentar, el proyecto CIAKL (Cinema and Industry Alliance for Knowledge and Learning) presenta una vía intermedia entre la formación presencial y la formación a distancia en la que la modalidad del curso no es un fin en si mismo, sino la búsqueda de la vía más apropiada para formar a un colectivo concreto en unas competencias concretas que responde a unas necesidades previamente identificadas. El proyecto CIAKL se desarrolla en el ámbito de las enseñanzas universitarias relacionadas con comunicación audiovisual a nivel europeo y está orientado a mejorar la formación de los estudiantes que cursan estas disciplinas en los ámbitos de la innovación y el emprendimiento para reducir el gap que pueda existir entre la formación académica y el desempeño profesional autónomo que les espera en un entorno laboral poco favorable.

\section{Metodología}

Una vez rematado el proyecto el 31 de julio de 2013 con la sesión final en Tallin, surge en los autores la idea de plasmar la experiencia de 19 meses trabajando en una iniciativa europea en el ámbito de las industrias culturales. De ahí que planteemos este trabajo como descripción y análisis crítico de una experiencia pionera en el campo de la formación en comunicación audiovisual y en la que participó la Universidad de Vigo a través de la Facultad de Ciencias Sociales y de la Comunicación. Habiendo formado parte del key staff, desglosamos en lo que sigue, por un lado, la fase previa al desarrollo del curso propiamente dicho y, por otro lado, la confección de módulos y sesiones de teledocencia incluidas en el proyecto, así como todas aquellas facetas inherentes a este tipo de proyectos como puede ser la selección de alumnos, su tutorización, etc.

Partimos de una observación in situ y desde dentro de todo el proceso a modo retrospectivo en el contexto tanto de la teledocencia como alternativa de futuro para iniciativas similares, como de la propia situación del sector audiovisual gallego y español en comparación con la situación de Portugal, Lituania, Hungría y Alemania, es decir, los demás países participantes, que generó un intercambio fructífero.

Fuentes manejadas son ante todo manuales y artículos de referencia en lo que a teledocencia y MOOCs se refiere, así como la propia documentación del proyecto CIAKL que ha servido de base para establecer la estructura, ya que debido a la especificidad del mismo no existe bibliografía específica al respecto. 


\section{Filosofía y objetivos}

La idea central del proyecto CIAKL surge de la necesidad de incorporar una serie de competencias orientadas a la innovación y al emprendimiento al currículo del alumnado de las titulaciones relacionadas con la industria audiovisual y multimedia. Por ello considerábamos que la puesta en contacto de las realidades formativas orientadas a la innovación de distintos países europeos, dotaría al proyecto de una perspectiva internacional imprescindible en el actual contexto económico. Además, la implicación activa de empresas del ámbito audiovisual y tecnológico contribuiría a desarrollar de un modo óptimo una serie de competencias que necesariamente se aplicarían fuera del ámbito estrictamente académico.

Así pues, los socios académicos e industriales trabajaron a lo largo del proyecto en el diseño y puesta en práctica de un plan formativo en el que tanto los contenidos como las metodologías estuviesen orientados a fortalecer competencias relacionadas con la innovación y el emprendimiento a través del trabajo colaborativo y el aprendizaje basado en proyectos.

El desarrollo del proyecto supuso dos niveles de trabajo con objetivos a corto y a medio-largo plazo. Por una parte, en el nivel más inmediato, se plantearon las necesidades para la puesta en marcha de un proyecto formativo que combinase las sesiones presenciales con la formación a distancia, el aprendizaje basado en proyectos (ABP) y la integración con las empresas, y, por otra, se establecieron objetivos a medio y largo plazo relacionados con la estandarización del modelo piloto y la creación de plataformas formativas estables.

En el desarrollo del presente artículo centraremos la reflexión en aquellas tareas relacionadas con el diseño de un currículo formativo común en el que primasen las competencias orientadas al emprendimiento y en el desarrollo y resultados de la aplicación de ese currículo en un curso especializado combinando la formación presencial y la teledocencia.

\section{El curso}

Las primeras decisiones con respecto al curso estuvieron relacionados con la concreción de contenidos en función de la necesidades formativas, en consonancia con el filosofía de trabajo con la que se diseñó la estrategia docente.

\subsection{Identificación de necesidades}

En el plan de estudios del Grado en Comunicación Audiovisual de la Universidad de Vigo se incluyen materias vinculadas con la gestión de empresas audiovisuales, sin embargo su carácter introductorio no le permite abordar cuestiones relacionadas con la operativa vinculada con el emprendimiento, como la internacionalización o la parte financiera. Desde la especificidad de cada uno de los socios del proyecto 
y atendiendo a sus objetivos, se estructura un trabajo conjunto para la definición de los contenidos del curso, que habrían de sustentarse en cuatro pilares básicos: las industrias culturales en el contexto europeo, el desarrollo de una idea emprendedora, la publicitación de la idea emprendedora y las formas de financiación, planes de negocio y viabilidad.

La Universidad Lusófona de Lisboa coordinó el diseño, desarrollo y calendarización de los contenidos y junto con el resto de universidades participantes en el proyecto, elaboró el programa docente que se expone a continuación.

\subsection{El diseño de contenidos}

El curso se diseñó con el objetivo de que el inicio y desarrollo de los contenidos fuese el mismo para todos los centros, de forma que las sesiones conjuntas por videoconferencia encajasen de la misma forma dentro del calendario formativo. El curso se estructuró en dos semestres; uno primero de mayor carga teórica y un segundo semestre fundamentalmente dirigido a la implementación de la idea emprendedora en el tejido industrial a través de la creación de un contexto profesional controlado académicamente.

Durante el primer semestre, las sesiones presenciales respondían a una duración de tres horas y media, con una cadencia semanal. La estructura en dos partes respondían a la celebración de una sesión online de media hora de duración a través de la plataforma WebEx y el desarrollo de un módulo a continuación por un período de tres horas. Las sesiones online fueron llevadas a cabo por integrantes de las empresas colaboradoras en el proyecto, muchas de ellas multinacionales, que pudieron transmitir un enfoque global a propósito del cambio de modelo productivo audiovisual y la reconfiguración del negocio vinculado a las industrias culturales en Europa. Este primer semestre se estructuró en 15 módulos más una reunión con la participación de las cinco universidades europeas, que se desplazaron a las instalaciones CISCO más cercanas para la celebración de una videoconferencia conjunta el 25 de febrero de 2013. En este sesión, que cerraba el bloque más teórico del curso, cada socio presentó su proyecto y exhibió un material audiovisual, en forma de piezas de 3 minutos y 10 minutos de duración, que habrían de servir no solo para explicar el proyecto empresarial, sino también para buscar posibles fuentes de financiación.

Antes de la finalización de esta sesión conjunta se definieron los objetivos del segundo semestre, que habría de significar la implantación de la idea emprendedora en el tejido industrial con la colaboración de una empresa del sector, que pudiese acoger la idea de los alumnos y coordinar su desarrollo, permitiendo el uso de sus instalaciones y el asesoramiento de sus profesionales.

4.3 Metodología y herramientas.

Zabalza (2011: 88) define las metodologías docentes a través de cuatro dimensiones básicas: la organización de los espacios y los tiempos, el modo de suministro de 
la información, la orientación y gestión de las actividades de aprendizaje y las relaciones interpersonales. La configuración de cada una de ellas se articulará teniendo en cuenta las características de las instituciones académicas, las cualidades humanas de los individuos que las integran y los recursos disponibles vienen a dar definir y condicionar la elección de la metodología docente. En el caso del proyecto CIAKL este segundo grupo de factores exógenos determinan el diseño de la estrategia en la medida en que debía ser compartida por distintos países europeos, respondiendo a un alto grado de compatibilidad en cuanto a recursos disponibles, necesidades formativas y dinámicas de enseñanzas locales, amén de las diferencias de carácter cultural que pudieran intervenir, complicando y enriqueciendo el proceso.

Así, para dar respuesta al currículo formativo se toma la decisión de implementar tres metodologías docentes, cuya importancia se fue amoldando a las necesidades estructurales del curso. Se trataba por tanto de establecer una estrategia más en relación con las fases del proceso de aprendizaje que con los contenidos, coincidiendo con Zabalza (2011: 97), que invita a una combinación de metodologías en este sentido, atendiendo también a las funciones de aprendizaje. Las metodologías docentes escogidas fueron: lecciones magistrales (presenciales o través de conferencia), trabajo autónomo y en grupo y aprendizaje basado en proyectos (ABP).

La lección magistral como metodología exclusiva dentro del proyecto presentaba limitaciones, fundamente relacionadas con el carácter práctico del curso. Además, el reducido número de alumnos nos permitía la implementación de una estrategia más cercana al trabajo profesional, el ABP. Se trataba de buscar una solución metodológica en consonancia con las reflexiones de Arques, Aznar, Carbonell et al. (2006) a propósito del aprendizaje en contexto de creación colectiva. Los autores abogan por el ABP como método de aprendizaje transversal, alejándose de la compartimentación del conocimiento en diferentes materias, la lección magistral como medio principal y el estudio individual y el examen como herramienta de evaluación. En esencia se trata de un aprendizaje que ocurre durante la aplicación del conocimiento a casos reales a través del trabajo en grupo.

En la orientación del curso, centrada en el fomento del emprendimiento desde una perspectiva académica, el ABP en combinación con seminarios presenciales y conferencias a través de sistemas telemáticos constituyeron las herramientas clave para mejorar las competencias de los alumnos. Al mismo tiempo, las estrategias docentes debían ser capaces de asimilar la participación de los demás socios del proyecto, a través de sesiones de carácter teórico compartidas. En esencia se trataba de reforzar las lecciones magistrales y el ABP a través de recursos de teledocencia.

Las sesiones de videoconferencia con profesionales europeos se realizaron a través del sistema Webex y la plataforma Quad, herramientas desarrollados por CISCO, socio del proyecto. Quad ofrecía también su funcionalidad como red social facilitando la creación de debates e intercambio de fuentes de información, así como la posibilidad de disponer de un repositorio de contenidos complementarios a los desarrollados por los docentes y profesionales participante en el curso. Más espe- 
cíficamente diseñada para la gestión de proyectos colaborativos en línea, se utilizó la herramienta Basecamp, que permitió la visualización del proceso de evolución de la idea emprendedora, coordinar reuniones, videoconferencias y funcionar como punto de encuentro virtual entre los coordinadores del proyecto por la Universidad de Vigo y los socios profesionales. Finalmente, la plataforma online para la distribución de videos Show and Share, desarrollada también por CISCO, facilitó la gestión del material audiovisual generado por todos los socios del CIAKL.

\subsection{Implementación del programa docente}

La formación teórica y sus posibilidades de aplicación práctica se estructuraron en quince módulos semanales que abordaron los siguientes temas: emprendimiento y gestación de ideas, el estado actual y claves de futuro del mercado del entretenimiento, la convergencia digital, la cadena de valor en las industrias culturales, los derechos de autor y la presentación de proyectos, módulo que corrió a cargo de la Universidade de Vigo y que se impartió online para el resto de las universidades participantes en el proyecto. Además, esta sesión sirvió para introducir la parte más económico-financiera centrada en el desarrollo del plan de negocio. Para finalizar, se desarrollaron dos módulos sobre marketing estratégico y operacional y otro sobre plan de producción.

Completados los módulos, los alumnos prepararon una presentación de su idea de startup, que se concretó en un video breve, de tres minutos de duración, que se compartió con el resto de universidades con el objetivo de recibir comentarios y sugerencias a propósito de cada una de las propuestas. Ello dio lugar a un proceso de implementación de mejoras que coincidió con las vacaciones de Navidad y se extendió el mes de enero, de forma que en febrero se pudiese entregar el plan de empresa actualizado junto con un video de 10 minutos de duración en el que se desarrollaban cuestiones relativas al plan de negocio y viabilidad. De esta forma se celebra la sesión que daría por concluido el primer semestre y en la que el proyecto "Creactivos" es destacada como la segunda mejor idea emprendedora del proyecto CIAKL. Se daba paso por tanto a la implementación del proyecto en el tejido empresarial que habría de concluir el 10 de julio del 2013 con la reunión final de todos los participantes.

El trabajo de coordinación del proyecto durante el segundo semestre estaba condicionado por la elección de un tutor externo en forma de profesional del sector y un socio, en forma de empresa, cuyas actividades estuviesen relacionada con la iniciativa empresarial de la Universidad de Vigo "Creactivos", que se desarrolla específicamente en el siguiente apartado. Se trataba de gestionar el progreso de la idea y su concreción en un proyecto real, otorgando tanta importancia al resultado como al proceso en si mismo. La labor de coordinación del proyecto CIAKL por parte de la Universidad de Vigo y del proyecto "Creactivos" por parte de la empresa A Navalla Suiza, permitió crear un tejido colaborativo que llevó la idea hasta su presentación final en Tallin, donde fueron invitados representantes del sector audiovisual y de la comunicación a nivel europeo, así como una delegación de la Comisión Europea correspondiente a este proyecto. 


\section{De la idea al proyecto "Creactivos".}

Desde nuestro punto de vista, la idea emprendedora debía generarse desde el propio alumnado participante. Para propiciar la creación de un contexto adecuado de creación colectiva, se implementa a las sesiones teóricas un espacio de colaboración creativa, en las que un experimentado emprendedor en el ámbito de las industrias culturales guía a los alumnos en el proceso de definición de su idea de negocio.

En un contexto como el actual, definido en gran parte por un retraimiento de la economía global y un pesimismo generalizado, la búsqueda de la idea se desarrolla en la línea de una visión más amplia del contexto, focalizándose fundamentalmente en las oportunidades que surgen derivadas del cambio de modelo productivo audiovisual y en la revalorización de la idea por encima de los recursos disponibles. Así nace "Creactivos", una idea de negocio en la que los alumnos son, al mismo tiempo, emprendedores y público objetivo.

"Creactivos" es una herramienta web para canalizar y visibilizar las competencias de los estudiantes de Comunicación Audiovisual mediante la distribución y gestión de sus trabajos audiovisuales. A través de la página web se ofrecen también otros servicios como la formación, un sistema de valoración de los contenidos audiovisuales gestionado por expertos del sector y servicios de facturación para profesionales que no estén dados de alta en la seguridad social. Todo ello pretende crear una red colaborativa para la puesta en valor del talento de aquellos alumnos que no han tenido la oportunidad de acceder al mercado laboral y poder acreditar sus habilidades. Con ello se facilita la adquisición de experiencia demostrable, en un sector en el que mostrar el trabajo realizado resulta un factor de selección de personal de primer orden.

La definición de la idea emprendedora permite articular por un lado las necesidades formativas asociadas al proyecto y por otro, la propia estructura del curso ya predefinida. Así se define una metodología docente basada en proyectos, desarrollada a partir de estrategias de aprendizaje flexibles en las que la colaboración entre docentes y profesionales del sector resulta fundamental. Se trata en definitiva de una búsqueda por sentar las fundamentos logísticos de un tejido colaborativo entre la universidad y la empresa, en consonancia con algunas de la ideas que han inspirado el Espacio Europeo de Educación Superior.

\subsection{El desarrollo del proyecto "Creactivos"}

La concreción de "Creactivos" en cuanto a su área geográfica de actuación, target y funcionalidades de la página web, marca el inicio del curso, en tanto en cuanto sentará las bases para las posteriores fases del proyecto CIAKL. Éstas incluirán la realización de un plan de empresa, que formara parte de un primer informe que será remitido a la Universidad Lusófona de Lisboa, líder del proyecto. Así, en una reunión celebrada el 21 de marzo de 2013, las ideas y planes de negocio presentados por todas las universidades participantes se someten a un proceso de evaluación en el que se tendrá en cuenta no sólo la originalidad y atractivo de la idea, sino también su viabi- 
lidad de implantación y su capacidad de respuesta con respecto a los objetivos originales del proyecto europeo. Como resultado de la evaluación conjunta, "Creactivos" es instada, con el resto de las propuestas, a realizar una serie de mejoras y revisiones destinadas mejorar la competitividad del proyecto.

Durante el segundo semestre del curso y para la implementación de "Creactivos" en un entorno profesional, se integra a la empresa A Navalla Suiza en el equipo de la Universidad de Vigo. A partir de ese momento y durante los siguientes cuatro meses el trabajo de los alumnos se centra en la monetización de la idea y el desarrollo de la página, atendiendo a aspectos como la arquitectura de la información, el diseño y la interactividad. El progreso en el proyecto fue monitorizado por el key staff del proyecto a través de una estrecha comunicación con los socios profesionales, el uso las plataformas de trabajo colaborativo y la realización de videoconferencias en momentos clave del desarrollo de la idea empresarial. El trabajo de coordinación se centraba en esta parte del desarrollo del proyecto en no olvidar que las claves del éxito no residían sólo en los resultados obtenidos, sino también en el propio proceso de desarrollo del la idea como fuente de conocimiento y experiencia para los alumnos.

Así, el 8 de julio del 2013 se celebra en Tallin la puesta en común de resultados con todas las universidades europeas participantes. Durante las presentaciones se evaluarán las ideas emprendedoras y sus planes de empresa, así como la solidez del proyecto como modelo de aprendizaje. Con posterioridad a la celebración de esta reunión, se invita a los participantes a desarrollar un proceso de autoevaluación, que habrá de formar parte del informe final conjunto que se remite a la Comisión Europea, constituyendo una importante fuente de información para la Universidad de Vigo y los participantes en el proyecto.

\section{Conclusiones}

En primer instancia el proyecto CIAKL ha supuesto la creación de un tejido colaborativo entre universidades a través de un trabajo docente conjunto adaptado al marco normativo de cada uno de los centros y países participantes. Este hecho ha conllevado la creación de un valioso sustrato, un marco de referencia sobre el que poder desarrollar futuros proyectos de forma más eficiente y coordinada.

En este tejido colaborativo de ámbito europeo, la teledocencia está llamada a ocupar un lugar importante, como complemento a los programas formativos ordinarios y como canal habitual de intercambio cultural y de conocimientos. En este sentido, facilita también la normalización en el uso del inglés como idioma de docencia en países como España, en los que su uso es, aun a día de hoy y a pesar de los esfuerzos realizados por lo centros, excepcional.

En cuanto al desarrollo de "Creactivos", se ha desarrollado la página web atendiendo a la arquitectura de la información, el mapa del sitio, el diseño grafico y el 
planteamiento de la fase de interactividad, si bien su ejecución no fue posible por la exigencia técnica que requería y lo ajustado del calendario. Todo ello permitió a graduados con comunicación audiovisual adquirir nuevos conocimientos y poner en práctica las competencias adquiridas en un contexto alejado de los modelos tradicionales de producción audiovisual como el cine o la televisión. El proceso de reelaboración de la idea inicial y la asimilación de estrategias de marketing y emprendimiento ha dotado de los alumnos de una perspectiva más amplia del mercado laboral y ha potenciado los conocimientos adquiridos durante su formación al tener que adaptarlos a un nuevo contexto competitivo.

De los datos extraídos del sistema de autoevalución que forma parte del proyecto, identificamos dos valores que han sido resaltados por los propios alumnos; en primer lugar su participación ha mejorado sus habilidades comunicativas en contextos de creación colectiva y en segundo lugar se consideran autosuficientes en lo que se refiere a la búsqueda de recursos y fuentes de información necesarias para poner en marcha una iniciativa emprendedora. Por otro lado, los alumnos sugieren una vía de mejora a través de la creación de equipos de trabajo multidisciplinares, que les permita superar las dificultades desde el núcleo equipo de trabajo, fortaleciendo el tejido colaborativo y dando lugar a un aprendizaje constante.

El aprendizaje que ha supuesto a nivel académico y la experiencia que ha conllevado a nivel institucional nos lleva a incidir en la importancia de la puesta en marcha de este tipo de proyectos, ahora más que nunca, en un momento en la volatilidad del contexto tecnológico y la importancia de la vinculación universidad y empresa demuestran una dialéctica enriquecedora para ambas partes. Las enseñanzas potenciadoras de la creación de autoempleo y la investigación académica dirigida a nuevos modelos productivos audiovisuales nos llevan a desear formar parte de nuevos proyectos similares.

\section{Referencias bibliográficas}

ARQUES CORRALES, P., AZNAR GREGORI, F., CARBONELL SOTO, L. et al. (2006). Nuevas metodologías docentes ante el EEES: Aprendizaje Basado en Proyectos y su implementación con tecnologías para el trabajo colaborativo (Informe final de proyecto de investigación). Disponible en: http://www.i3a.ua.es/ fich_proyectos/2_6_Informe_ABP_definitivo.pdf. [05/09/2013]

SCOPEO (2013). "SCOPPEO INFORME N². MOOC: Estado de la situación actual, posibilidades, retos y futuro". En Scopeo Informe No. 2. Disponible en: http:// scopeo.usal.es/wp-content/uploads/2013/06/scopeoi002.pdf [09/09/2013]

ZABALZA BERAZA, M. A. (2011). "El Espacio Europeo de Educación Superior ¿Hacia dónde va la Universidad Europea?". En REDU. Revista de Docencia Universitaria, vol 9, $\mathrm{n}^{\circ}$ 3. Santiago de Compostela: Red Estatal de Docencia Universitaria. p. 75-98. Disponible en: http://red-u.net/redu/index.php/REDU/ article/view/302/pdf. [10/09/2013] 


\section{Notas}

$1 \mathrm{http} / / /$ davecormier.com/edblog/2008/10/02/the-cck08-mooc-connectivism-course-14-way

2 http://infocult.typepad.com/infocult/2008/07/connectivism-course-draws-night-or-behold-the-mooc. html

3 Coursera, una de las plaformas de MOOCs más nombradas tiene más de cinco millones de alumnos inscritos en los más de 450 cursos que ofrecen a través de la plataforma 90 universidades de todo el mundo. Comenzó a funcionar a finales de 2011. Además de esta plataforma existen otras muchas como Udacity, EdX o MiríadaX, promovida desde España por Universia, Santander y Telefónica.

\section{Los autores}

Juan Manuel Corbacho Valencia es Licenciado en Publicidad y Relaciones Públicas y Traducción e Interpretación por la Universidad de Vigo. Realizó su tesis sobre publicidad internacional, materia que imparte en la Facultad de Ciencias Sociales y de la Comunicación (Universidad de Vigo - Campus de Pontevedra) de la que en el momento de la publicación es decano tras pasar por otros cargos académicos como el de vicedecano de relaciones internacionales. Junto con su línea de investigación de comunicación publicitaria global, también ha publicado trabajos en el ámbito de las relaciones públicas y protocolo. Ha realizado estancias de investigación en Brasil, Chile, México, Portugal y Alemania.

Oswaldo García es Doctor en Comunicación Audiovisual por la Universidad de Santiago de Compostela, ha realizado estancias de investigación en la Universidad Lusofona de Lisboa (Portugal), Universidad Federal de Santa Catarina (Brasil) y Universidad de Costa Rica. Es realizador con más de diez años de experiencia en el sector audiovisual. Ha trabajado en productoras privadas y emisoras autonómicas realizando programas deportivos, infantiles, magazines y de ficción. Como trabajador autónomo ha participado en la realización de videoclips, acciones de videomapping, videoinstalaciones para artistas plásticos y la construcción de espacios sonoros.

Alberto Dafonte es Doctor en Publicidad y RR.PP. por la Universidad de Vigo con la tesis titulada Creación de identidad de marca en contenidos televisivos: aplicabilidad de modelos teóricos a los formatos de entretenimiento en televisión. Es Licenciado en Comunicación Audiovisual y Licenciado en Publicidad y RR. PP. Actualmente es profesor a tiempo completo en la Facultad de Ciencias Sociales y de la Comunicación de la Universidad de Vigo, en la que imparte las materias Teoría y práctica de la comunicación televisiva y Estrategias publicitarias y de relaciones públicas para productos audiovisuales. Sus líneas de investigación principales están ligadas a estos ámbitos. Es miembro fundador y vicepresidente de la asociación de profesionales de la publicidad en Galicia Creatividade Galega. 